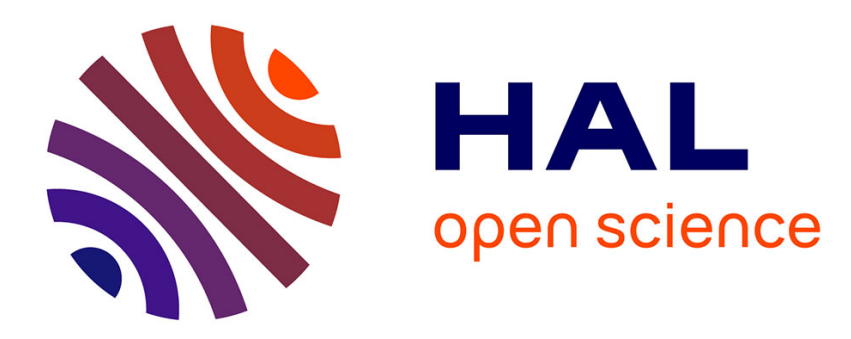

\title{
Valorisation des expériences en milieu professionnel dans la formation initiale
}

Hélène Veyrac, Nina Asloum

\section{To cite this version:}

Hélène Veyrac, Nina Asloum. Valorisation des expériences en milieu professionnel dans la formation initiale. J.F. Marcel; P. Olry. Recherches en éducation Pratiques et apprentissages professionnels, Educagri éditions, pp.145-156, 2014, 978-2-84444-987-0. hal-01558072

\section{HAL Id: hal-01558072 \\ https://hal.science/hal-01558072}

Submitted on 7 Jul 2017

HAL is a multi-disciplinary open access archive for the deposit and dissemination of scientific research documents, whether they are published or not. The documents may come from teaching and research institutions in France or abroad, or from public or private research centers.
L'archive ouverte pluridisciplinaire HAL, est destinée au dépôt et à la diffusion de documents scientifiques de niveau recherche, publiés ou non, émanant des établissements d'enseignement et de recherche français ou étrangers, des laboratoires publics ou privés. 
Veyrac, H., \& Asloum, N. (2014). Valorisation des expériences en milieu professionnel dans la formation initiale. Dans J.-F. Marcel \& P. Olry (Éds), Recherches en éducation: pratiques et apprentissages professionnels (pp. 145-156). Dijon : Éducagri éditions.

\title{
Valorisation des expériences en milieu professionnel dans la formation initiale
}

\author{
Helene VEYRAC, Nina ASLOUM \\ Université de Toulouse \\ Ecole Nationale de Formation Agronomique \\ UMR Education Formation Travail Savoirs \\ 2 Route de Narbonne BP 22687 \\ 31326 CASTANET-TOLOSAN Cedex FRANCE
}

\begin{abstract}
Résumé
Un ensemble de recherches sur le travail des enseignants de l'enseignement agricole public français a porté sur la valorisation des expériences des apprenants (apprentis ou élèves) dans leur formation. Les résultats de ces recherches permettent de mieux comprendre les pratiques d'accompagnement proposées par les enseignants pour le que "le travail permette d'apprendre". Ils portent d'une part sur ce que sont, pour les formateurs, les conditions pour que les situations pré-professionnelles de stage des apprenants permettent un apprentissage professionnel et d'autre part sur ce que les formateurs mettent en œuvre pour favoriser cet apprentissage.
\end{abstract}

Les travaux présentés dans ce chapitre s'intéressent aux pratiques des enseignants exerçant en formation professionnelle initiale. L'immersion des élèves en situation professionnelle et la valorisation de leurs expériences dans les parcours de formation constituent des thématiques récurrentes dans les débats concernant ces formations.

Plusieurs de nos travaux (notamment Bouillier, Asloum \& Veyrac, 2008 ; Veyrac, Asloum, 2009) ont participé à une meilleure compréhension de la valorisation des expériences en milieu professionnel dans la formation initiale de l'enseignement agricole. Ces travaux reposent sur l'analyse du travail des professeurs. Ils portent sur les activités des enseignants dans des dispositifs de formation alternant séquences en milieu professionnel et séquences en centre de formation. Ce chapitre est consacré à la présentation synthétique de ces recherches. 


\section{Table des matières}

1. La question de la fonction de l'expérience pour le développement.............................................. 3

2. Le métier d'enseignant en aménagement paysager.................................................................. 4

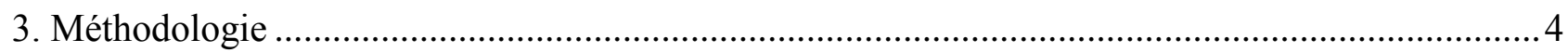

3.1. La mise en œuvre des situations didactiques ............................................................ 4

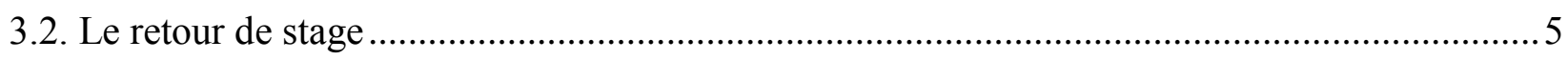

4. Selon les formateurs quelles sont les conditions pour que le travail permette aux apprenants

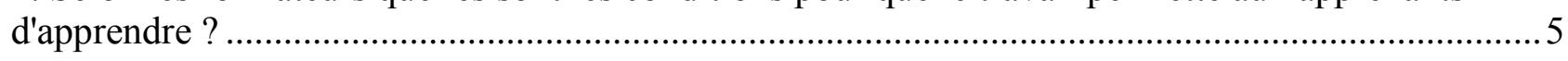

4.1 La progression pédagogique planifiée par l'enseignant...................................................... 5

4.2. Les quatre conditions pour apprendre des situations de travail ............................................ 6

4.2.1.L'auto-questionnement par rapport à une situation problème ........................................ 6

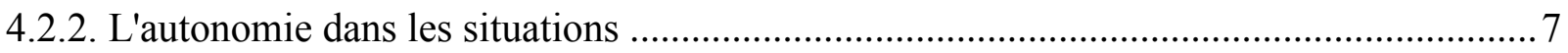

4.2.3. La nouveauté de la situation pour l'élève ..............................................................

4.2.4. Estimation positive des actions réalisées .............................................................

5. Quelles activités les enseignants mettent-ils en œuvre pour que le travail permette aux apprenants

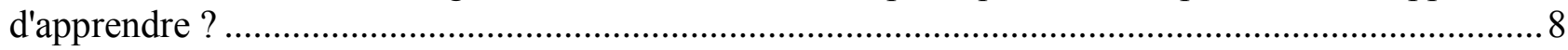

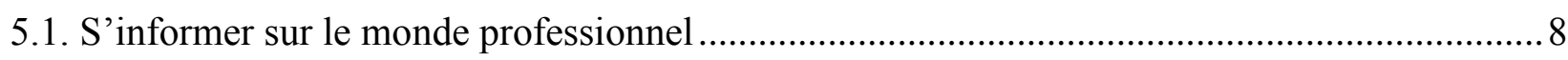

5.2. Choisir un référent parmi un ensemble de référents possibles............................................. 8

5.3. Adopter une stratégie didactique pour répondre aux écarts entre monde professionnel et

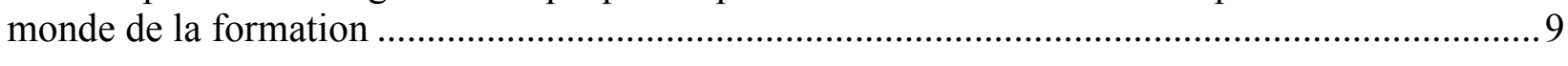

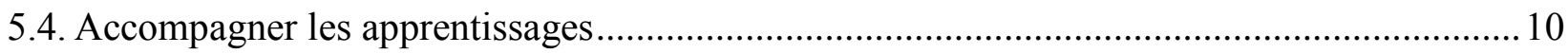

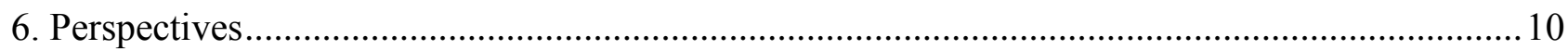

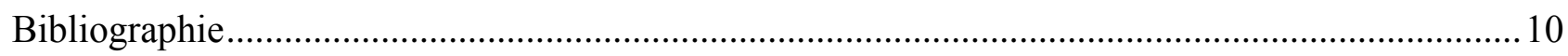




\section{La question de la fonction de l'expérience pour le développement}

Les dispositifs d'alternance semblent reposer sur le principe que les situations professionnelles permettent d'apprendre. Ce postulat a déjà été discuté (Mayen, 2007) : "quand on veut résoudre un problème de professionalisation, on convoque l'alternance et l'expérience et on ajoute un peu plus de temps d'expérience et un peu plus de temps d'encadrement pour des expérimentés. Si l'on était un peu sévère, on pourrait penser que la mise en situation professionnelle est une manière de résoudre hâtivement deux difficultés récurrentes de la formation professionnelle. La première est celle de la définition de ce qui est visé [...] La deuxième est celle de la "problématique de l'apprentissage orienté vers l'agir" (Vanhulle, Merhan \& Ronveaux, 2007) ou, en terme de didactique professionnelle (Pastré, 1999) : comment apprendre des situations ?" (op cit, p.85).

L'ergonomie de l'activité pose, dans sa définition même, le postulat du développement par le travail. En effet, en définissant l'activité comme "ce qu'un professionnel met en œuvre dans un contexte de tâche et un contexte personnel particuliers pour produire et se développer " (Veyrac, Bouillier, 2011), l'activité comporte intrinsèquement une dimension "productive" et une dimension "constructive". Cette dernière dimension renvoie au développement. Poser que l'activité produit du développement ne permet cependant pas d'en préciser la nature. La didactique professionnelle apporte des éléments de réponse à cette question, notamment en tentant de déterminer les conditions du développement. Cet enjeu de recherche n'est pas le seul de la didactique professionnelle ; il n'est pas non plus la propriété exclusive de la didactique professionnelle tant la question est centrale pour nombreuses recherches notamment en psychologie du développement et pour la clinique de l'activité.

L'approche que nous défendons est une approche originale puisqu'il ne s'agit pas de donner notre avis de chercheur sur la question des conditions du développement professionnel, mais de rechercher des réponses par le biais des théorèmes en actes "de professionnels de l'alternance". Les professionnels retenus sont des formateurs en aménagement paysager en charge d'aider des apprenants à se préparer à une vie professionnelle dans ce domaine.

Une première partie du chapitre reprendra les résultats d'une recherche conduite auprès d'un enseignant dont l'analyse de l'activité a fait l'objet d'un chapitre d'ouvrage (Bouillier, Asloum \& Veyrac, 2008), et d'un article (Veyrac, Asloum, 2009). Les entretiens d'auto-confrontation sur des séances de formation correspondant à des retours de périodes de stage des élèves ont permis de mettre en évidence le modèle de ce formateur guidant l'action de l'enseignant durant ces séances. Pour lui, dans quelles conditions le travail permet-il d'apprendre?

La deuxième partie rendra compte de diverses analyses de l'activité d'enseignants dans des dispositifs visant des apprentissages en situation de travail. C'est sous l'angle de l'activité des enseignants qu'est abordé la question des conditions qui permettent au travail d'apprendre : qu'est-ce qui est mise en œuvre par les enseignants pour favoriser le lien entre travail et apprentissage.

Enfin, une dernière partie définit les perspectives de recherche dans le prolongement des travaux présentés. 


\section{Le métier d'enseignant en aménagement paysager}

L'ensemble des résultats présentés reposent sur l'analyse du travail d'enseignants intervenants en sciences et techniques des aménagements paysagers. Ces enseignants sont recrutés au sein du ministère de l'agriculture dans le secteur de «l'aménagement de l'espace et protection de l'environnement». L'année de création de ce secteur dans l'enseignement agricole est relativement récente. Elle remonte aux années 1990. Elle correspond à une prise en compte de la gestion de l'environnement et à l'émergence d'une demande sociale concernant la qualité des paysages. C'est également à cette période qu'émerge de nouveaux métiers et de nouvelles pratiques professionnelles. Nous présenterons ici un aperçu du champ d'activités que recouvrent les métiers du secteur de l'aménagement paysager. Les composantes du secteur regroupe quatre familles de métiers dont les activités professionnelles sont liées à la forêt, aux travaux paysagers, à l'aménagement de l'espace et à la gestion et maîtrise de l'eau. Les aménagements paysagers recouvrent la création d'espaces paysagers très diversifiés. Ces espaces sont consacrés à la jouissance d'une nature aménagée, comme le jardin particulier, les parcs de loisirs, les jardins historiques ou dédiés à l'embellissement du cadre de vie de l'espace vert urbain, des infrastructures routières, des rivières... Ce secteur d'activités concerne la mise en place d'aménagements, depuis leur programmation et leur conception jusqu'à leur réalisation et leur gestion avec une prise en compte de la demande sociale croissante en matière d'environnement.

\section{Méthodologie}

Les analyses présentées infra ont pour origine la fin d'une recherche action qui portait sur les difficultés des enseignants à intégrer l'interdisciplinarité dans leur pratique quotidienne (Bouillier-Oudot, \& Asloum, 2004) et sur le travail collectif imposé par les formations interdisciplinaires (Asloum, \& Bouillier-Oudot, 2007). Cette recherche action s'est déroulée sur deux années, elle impliquait cinq équipes d'enseignants de différents établissements préparant au Brevet de Technicien Supérieur Agricole (BTSA) en aménagement paysager. Ce diplôme, délivré par le ministère de l'agriculture français, est préparé en deux années après le baccalauréat. Deux établissements ont été retenus sur la base du volontariat et de l'engagement dans la poursuite de l'étude. La première partie de nos résultats s'appuie sur des données constituées des commentaires produits par un enseignant lors d'une autoconfrontation. Cet enseignant intervient en BTSA par alternance. La deuxième partie s'appuie sur ce même recueil de données, mais également sur un recueil effectué dans un deuxième établissement auprès d'enseignants intervenants dans la même filière de formation.

Les méthodes employées pour recueillir les données varient selon les types de situations observées.

\subsection{La mise en œuvre des situations didactiques}

Un entretien semi-directif, mené par les mêmes chercheurs dans les deux établissements, a été enregistré et retranscrit. II s'agissait d'une présentation par les enseignants de l'ensemble du dispositif de formation. Les entretiens se sont centrés sur les choix et la mise en œuvre des principales situations didactiques visant l'acquisition de compétences professionnelles. 


\subsection{Le retour de stage}

La séance d'enseignement concernant les retours de stage a eu lieu quatre mois après le premier enregistrement évoqué précédemment.

- Cette séance, d'une durée de deux heures, a été filmée à l'aide d'une mini-caméra cravate. Cette caméra «subjective» a permis d'une part, de ne pas confronter l'enseignant à sa propre image, et d'autre part, d'enregistrer les prises d'informations visuelles et auditives de l'enseignant.

- L'autoconfrontation simple a également été filmée. Pour ce faire, une caméra enregistrait la télévision qui diffusait le film issu de la caméra subjective et les commentaires de l'enseignant. L'autoconfrontation s'est déroulée à la suite de deux séquences "retour de stage". Chacune d'elles ont duré deux heures. Les échanges pendant le visionnement des films ont été retranscrits.

\section{Selon les formateurs quelles sont les conditions pour que le travail permette aux apprenants d'apprendre?}

Dans cette partie, nous nous intéresserons aux modèles qui fondent les pratiques des enseignants dans les séances de retour de stage : dans quelles conditions le travail permet-il d'apprendre selon l'enseignant en filière professionnelle ? II s'agit de décrire les conditions utiles ou nécessaires pour l'apprentissage par le travail, du point de vue des formateurs.

Cet enseignant intervenant dans un dispositif de formation par alternance considère que les situations professionnelles vécues en stage par ses élèves sont un moyen d'apprentissage inachevé. L'apprentissage ne peut se constituer sans l'étayage de l'école. C'est à l'enseignant et de manière différée, que revient la prise en charge de la conceptualisation des actions. Ainsi, pour l'enseignant, la situation de stage ne peut pas, à elle seule, être une condition suffisante pour apprendre. Au-delà, de l'immersion dans une situation professionnelle, le travail permet d'apprendre si certaines autres conditions sont réunies.

Avant de présenter ces conditions, nous précisons dans quel dispositif didactique s'intègre le déroulement des séances, dispositif qui traduit - partiellement - la prise en charge par l'école de conditions pour favoriser l'apprentissage. En effet, la mise en place de ce dispositif et la manière dont il est structuré, donnent des indications concernant les conditions que le formateur tient pour nécessaires à l'apprentissage par le travail.

\subsection{La progression pédagogique planifiée par l'enseignant}

L'enseignant tend à ajuster les contenus proposés en formation sur la base d'activités réalisées par les élèves en entreprise. Pour ce faire, l'enseignant utilise un logiciel d'e-portfolio dans lequel sont décrites les tâches et les activités significatives du métier (les situations professionnelles faisant partie du cœur de métier). Cette description a été réalisée par l'enseignant avec la participation des maîtres d'apprentissage. Comme nous le faisions remarquer préalablement, le secteur d'activités des entreprises en aménagement est très varié et présente une grande diversité de situations professionnelles qui ne sont pas forcément couvertes par l'ensemble des entreprises dans lesquels les élèves effectuent leurs stages. Par conséquent, chaque élève, ne pourra pas systématiquement rencontrer l'ensemble des tâches prescrites. Grâce à l'utilisation de l'e-portfolio, les tâches rencontrées par les apprenants peuvent être régulièrement vérifiées par l'enseignant et le maître d'apprentissage. Cette prise d'information permet à l'enseignant, de construire une progression en intégrant cinq projets interdisciplinaires dont deux sont définis 
avec les maîtres de stage en fonction des besoins des élèves, un troisième que l'enseignant programme systématiquement sur des études préalables paysagères et enfin les deux derniers sont réalisés en fonction d'opportunités d'appels d'offres que les entreprises cèdent à l'école. En prenant en compte le " manque » tangible de l'entreprise, l'enseignant va donc définir le contenu de deux projets à partir des besoins des élèves recensés à partir de l'e-portfolio, un troisième projet pluridisciplinaire d'une semaine est consacré aux études paysagères d'un espace. L'étude préalable repose sur des études d'impact et d'environnement. Ces études complexes sont très rarement abordées dans les petites entreprises alors qu'elles font partie d'un des objectifs généraux de la formation. D'ailleurs, le référentiel du diplôme du brevet de technicien supérieur en aménagement paysager (DGER, 1994) consacre un module entier de $80 \mathrm{~h}$ intitulé «les techniques utiles à l'étude paysagère préalable à un aménagement». C'est un module technique que l'élève doit maîtriser, il s'agit de la première étape du processus d'aménagement : les études préalables. Le futur technicien sera appelé dans sa vie professionnelle à participer et à suivre les études préalables afin de mettre en œuvre, au sein d'un chantier, les techniques les plus appropriées. Ainsi dans cette formation technique et professionnelle l'enseignant s'astreint à couvrir l'ensemble du référentiel de formation. II construit non seulement des projets à partir des besoins des élèves mais aussi des études de cas qui ne seront jamais abordés dans les entreprises. Ces projets sont programmés dans le temps en suivant une certaine progressivité ; les deux premiers répondent aux besoins des élèves, le troisième sur l'étude préalable est proposé en fin de première année, lorsque les élèves sont en mesure de mobiliser les apports théoriques de la formation et les deux autres en deuxième année, au gré des opportunités professionnelles (appels d'offres) qui s'offrent à l'enseignant.

Par ailleurs, l'enseignant structure sa progression avec des activités en centre de formation et en entreprise selon quatre phases progressives sur les deux années de formation. En début du cycle de formation, les élèves sont invités à observer pour ensuite, dès le milieu de la première année, être à même de participer aux activités. La deuxième année, ils sont appelés à s'affranchir et montrer leur capacité à intervenir non seulement en parfaite autonomie mais aussi en faisant preuve d'une certaine expertise en fin d'année. Ce modèle dit "OPAE": Observer, Participer, être Autonome, exercer une Expertise, est prôné dans le cadre de la formation des enseignants intervenants dans les filières de formation initiale par apprentissage.

\subsection{Les quatre conditions pour apprendre des situations de travail}

L'analyse de l'entretien d'auto-confrontation a permis de dégager quatre conditions indépendantes pour apprendre des situations de travail selon l'enseignant observé.

\subsubsection{L'auto-questionnement par rapport à une situation problème}

Face à une situation problème, l'élève doit pouvoir, non seulement la résoudre mais surtout procéder à un auto-questionnement.

Lors de la séance de retour de stage, les élèves à tour de rôle énoncent les tâches réalisées en entreprise. Ils décrivent globalement les circonstances dans lesquels ils les ont réalisées. Le temps de restitution accordé à chaque élève ne permet pas de rentrer finement dans le déroulement des opérations. Ces séances sont réalisées, au dire de l'enseignant, afin de s'informer sur l'attitude manifestée par les élèves en situation de travail. S'est-il questionné ? Était-il en dans une situation problématique ? Cette situation l'a-t-elle amené non seulement à la résoudre, mais aussi à se questionner? 
L'enseignant nous clarifie sa position, en citant le cas d'une élève qui intervient dans un bureau d'étude en aménagement paysager. Son entreprise le charge d'établir des devis de travaux d'aménagements. Cette activité sollicite une parfaite connaissance des budgets de fonctionnement et d'investissement. Pour ce faire, les élèves doivent nécessairement disposer de connaissances de base dans le domaine des mathématiques. L'enseignant nous indique que l'élève est en situation d'échec et se trouve en position difficile vis à vis de son entreprise. Pour résoudre cette situation, l'enseignant souhaite que l'élève ressente la nécessité d'aller, de sa propre initiative, rechercher les ressources à mobiliser. Selon lui, répondre à l'exigence d'une situation de travail va contraindre l'élève à surmonter les obstacles qui dans ce cas, étaient d'ordre épistémique.

\subsubsection{L'autonomie dans les situations}

La maîtrise d'une tâche doit être confrontée à l'exercice d'une situation réelle, mais qui plus est, libérée de toute intervention; l'autonomie s'acquiert en faisant seul.

La séance analysée se situe à la fin de la première année et vise à préparer les élèves à un certain niveau d'autonomie de décision et d'action correspondant à la qualification d'un technicien supérieur. Cette phase est déterminante pour l'enseignant. Systématiquement, l'enseignant vérifie, auprès de chaque élève, leur niveau d'implication et leur niveau d'autonomie dans la réalisation des tâches. Pendant cette séance, les élèves s'expriment à l'oral individuellement ou en interaction avec les autres. Ces échanges lui permettent de mettre à jour les véritables acquisitions des élèves et de vérifier la véracité des propos tenus par les élèves à partir de leurs récits écrits.

Cependant, l'enseignant nous signale qu'un apprentissage réussi est soumis à la présence d'un tuteur. Cet accompagnement est rendu nécessaire par la nature même des situations problèmes pour lesquelles l'apprenant ne peut pas assumer seul le degré de difficulté, il faudra cependant l'amener à résoudre seul des problèmes.

\subsubsection{La nouveauté de la situation pour l'élève}

Pour apprendre, l'élève doit être confronté à une situation nouvelle et doit être en mesure de prendre des risques, même s'il ne maîtrise pas complètement la situation.

Selon l'enseignant, bien que certains élèves possèdent une expérience professionnelle antérieure, ils ne se mettent pas forcément en situation d'apprendre. En effet, il note que lorsque les entreprises proposent des activités intéressantes, si celles-ci ont déjà été réalisées par les élèves, ces derniers ne chercheront pas toujours à aller vers de nouvelles activités. Ils se cantonneront au champ d'activité proposé sans essayer d'aller vers des situations qu'ils ne maîtrisent pas. Pour lui, ces élèves auront moins de chance d'évoluer et de développer de nouvelles compétences.

\subsubsection{Estimation positive des actions réalisées}

L'enseignant cherche pendant cette séance à valoriser les progrès des élèves en manque de confiance. Certains élèves se retrouvent dans des situations professionnelles peu significatives du métier comme les tâches liées à l'entretien des espaces. Dans ce cas, ils ont le sentiment de ne rien apprendre en entreprise. L'enseignant incite les élèves à extraire des éléments de la situation leur permettant de valoriser l'activité de travail. II se saisit des expériences vécues par les élèves, même si celles-ci ne recouvrent qu'une petite partie des situations signifiantes du métier, comme levier pour provoquer chez les élèves en difficulté une estimation positive des activités réalisées. L'élève doit se sentir suffisamment sûr de lui face aux actions réalisées. 
On peut remarquer que les quatre conditions présentées relèvent des facteurs de variabilités inter-individuelles dans l'apprentissage des élèves. Plusieurs conditions à l'apprentissage professionnel énoncées par l'enseignant sont par ailleurs développées par la didactique professionnelle, notamment celles relatives au questionnement, à l'autonomie, à la tutelle, à la confrontation à des situations professionnelles par les élèves. De la même manière, les conditions ne se reportent pas exclusivement à l'apprenant. En effet, l'enseignant prend en charge certaines conditions de d'apprentissage (mise en place de séance de retour de stage, projet interdisciplinaire, logiciel de suivi des apprenants, etc.). On retrouve bien là les deux facteurs déterminants de l'apprentissage : des facteurs internes et des facteurs externes à l'activité d'apprentissage, tels que proposé par la psychologie ergonomique (Leplat, 1997), discipline dans laquelle la didactique professionnelle a puisé un cadre théorique structurant.

\section{Quelles activités les enseignants mettent-ils en œuvre pour que le travail permette aux apprenants d'apprendre ?}

Plusieurs activités d'enseignants peuvent être listées issues de nos recherches, il s'agit d'éléments complémentaires aux conditions précédemment dégagées, pour aborder la valorisation des expériences des apprenants en milieu professionnel.

\subsection{S'informer sur le monde professionnel}

Une activité mise en exergue par nos recherches est relative à la prise en compte, par les enseignants, du travail des professionnels. Cette prise en compte passe par une veille informationnelle aux sources multiples. Les échanges qui ont lieu lors des formations des personnes-relais (maître de stage, maître d'apprentissage, tuteur, etc.) sont une source informationnelle forte puisqu'elle peut donner à voir aux formateurs les préoccupations d'un ensemble de professionnels. Citons également les lectures de revues spécialisées, la participation à des manifestations professionnelles, la participation à la conception de référentiel professionnel (dans le cadre d'une conception ou d'une rénovation de diplôme) ou encore l'accompagnement des apprenants (visite de stage, consultation des e-portfolios, visionnage des films des apprenants, séance de retour de stage, etc). Ces situations sont autant de sources d'information pour les formateurs pour se représenter un métier. Ces information sont perçues par le prisme de la formation : c'est en tant que formateur, et non en tant que professionnel, que le formateur accède à cet ensemble d"information. II peut exister des formateurs "double-actifs" qui sont formateur et professionnel, ce qui modifie cette activité, mais nous n'avons pas rencontré ce cas pour les formateurs en aménagement paysager.

\subsection{Choisir un référent parmi un ensemble de référents possibles}

Les formateurs sont tenus de suivre des prescriptions leurs imposant des contenus de formation. Par ailleurs, ils sont sensés tenir compte du vécu des apprenants et préparer au mieux leur insertion professionnelle. Ces deux points ne s'opposent pas toujours, mais constituent parfois la base d'injonctions paradoxales (Veyrac, Bouillier, 2011), notamment lorsque les logiques du monde professionnel s'opposent aux logiques des référentiels de formation. Choisir un référent à enseigner parmi un ensemble de référents possibles est alors une activité nécessaire pour mener à bien leur travail de formateur. C'est l'analyse de la variabilité inter-individuelle de l'activité des formateurs qui nous a amenées à identifier cette activité de décision. Les choix sont parfois décidés implicitement, sans que les formateurs n'en aient toujours pleinement 
conscience. Nous avons pu mettre en évidence (Veyrac, Asloum, 2009) que les tâches professionnelles retenues par les formateurs comme devant être enseignées (« le référent à enseigner ») pouvaient se référer aux référentiels des diplômes (référentiels officiels) mais aussi aux activités professionnelles observées par les apprenants en stage en milieu de travail ou encore à des pratiques professionnelles innovantes. Ainsi est mis en évidence le choix par l'enseignant des situations de références, choix qui déterminent les situations retenues comme propices à l'apprentissage.

\subsection{Adopter une stratégie didactique pour répondre aux écarts entre monde professionnel et monde de la formation}

Certains enseignants peuvent être amenés à évoquer d'emblée aux apprenants l'existence d'un "décalage" entre ce qui est enseigné à l'école et ce qui est pratiqué en entreprise, comme pour couper court à toutes critiques possibles. Ils annoncent l'écart comme inhérent à leur propre métier d'enseignant en formation professionnelle.

Nous avons pu montrer (Veyrac, Asloum, 2009) que la perception des enseignants du travail des professionnels du monde agricole pouvait être basée sur le constat d'un écart entre les pratiques professionnelles qu'ils enseignent et celles que les professionnels mettent en œuvre. Nous avons évoqué la notion de "hiatus". Les attributions causales de ces hiatus sont multiples. En effet, les enseignants évoquent plusieurs origines à ces écarts, notamment les contraintes de rentabilité, de rapidité, auxquelles sont soumises les professionnels, l'évolution des techniques et de la demande sociale (durabilité, respect de l'environnement). Ils rejoignent ainsi Mayen (2007,p. 85) qui souligne également que l'exercice de l'activité en milieu professionnel "produit des activités approximatives, simplificatrices, marquées par la référence à des critères de réussites immédiats". Les enseignants peuvent se sentir désemparés face à ce constat de hiatus, de décalage. Des travaux (Chatigny \& Veyrac, 2005), ont évoqué l'existence de facteur de risque de détresse psychologique engendrée par cette tension entre les pratiques des professionnels des métiers auxquels les enseignants forment et les pratiques que se donnent à enseigner les enseignants.

D'autres enseignants adoptent des stratégies pour réduire le hiatus notamment en mettant en place les conditions d'émergence d'un discours des apprenants sur les pratiques effectives des professionnels. Nous avons ainsi décrit l'activité d'un enseignant exploitant les récits d'apprenants évoquant des pratiques professionnelles en décalage avec les pratiques professionnelles qu'un enseignant peut prôner. II s'agissait de pratiques illégales. Celles-ci étaient liées à des situations variées : le montage d'appel d'offre, l'absence de port de casque en situation professionnelle à risque, l'enfouissement de gravats à l'insu du client et le respect des délais de temps de séchage du ciment. Le parti pris de l'enseignant était de permettre aux apprenants d'évoquer ces situations professionnelles vécues. Sans les cautionner, il leurs laisse la place dans le centre de formation, une place circonscrite. Parfois, il en recherche les logiques par le questionnement avec les apprenants, dans une visée plus compréhensive que formatrice, tout au moins à court terme. Comprendre le travail effectif semble lui permettre de pouvoir mieux former, avec ou contre ces situations mises à jour, mais non niées, car bien présentes dans les expériences de vie des apprenants. Plusieurs buts de ce formateur ont ainsi été identifiées comme : chercher à dévoiler les pratiques dangereuses, déculpabiliser l'apprenant, s'appuyer sur l'effet de rejet des apprenants pour légitimer de futurs enseignements. 


\subsection{Accompagner les apprentissages}

Concernant les séances de retour de stage, nous avons pu plus précisément distinguer trois buts poursuivis par l'enseignant (1) identifier des demandes de formation des apprenants (2) valoriser les progrès des apprenants, les mettre en confiance (3) identifier les sur-estimations des apprenants. On peut remarquer qu'aucun discours d'enseignant n'a porté sur le rôle de la mise en mot de la situation vécue dans la construction de l'expérience. L'expérience qui consiste à revenir sur la situation vécue n'a pas été énoncée en tant que source de développement.

Concernant la visite de stage, nous avons pu mettre en évidence (Veyrac, 2005) différentes sources d'information privilégiées par les enseignants : les élèves, les maîtres de stage, les professionnels du monde agricole. Elles interviennent à différents temps de l'activité de suivi de stage : les réunions de préparation des stages, l'aide au choix des lieux de stage, les visites de stage, le travail de concertation en équipe éducative, les relations avec les parents, l'aide à la rédaction des rapports de stage, l'exploitation des stages en établissement d'enseignement. Elles sont liés aux but poursuivis par le formateur qui sont notamment (1) accéder à ce que l'apprenant apprend (2) accompagner l'apprentissage issu de l'analyse des situations professionnelles.

A l'issue de cet aperçu de plusieurs recherches, on peut avancer que l'analyse du travail des enseignants est une voie pour développer la didactique professionnelle, non seulement dans ces applications (eg. comprendre le travail des enseignants pour mieux les former) mais aussi dans l'identification des conditions nécessaires à l'apprentissage.

\section{Perspectives}

Ce chapitre s'est appuyé sur une revisite de recherches passées sur l'activité des enseignants relative à la valorisation des expériences en milieu professionnel. II montre l'intérêt de décrire le travail des formateurs pour la didactique professionnelle et au-delà pour la formation des formateurs et l'amélioration des formations professionnelles.

Ce travail initié est actuellement poursuivi sous plusieurs angles.

Le premier angle, dans la lignée des recherches précédentes, est celui de la description du modèle des professionnels de l'éducation relatifs à "l'objet apprenant". Comment un enseignant perçoit-il un élève ? Quels sont les traits saillants, en fonction de quoi se différencient-ils ? Le deuxième angle est celui de l'usage par les enseignants des vidéos des apprentis tournées en situation professionnelle : il s'agit également d'une continuité des recherches présentées dans ce chapitre. De plus, ces recherches se situent toutes dans le domaine de la recherche sur l'enseignement agricole.

\section{Bibliographie}

Asloum, N. \& Bouillier-Oudot, M.H. (2007). Pratiques collectives d'enseignants engagés dans une formation interdisciplinaire. Congrès international AREF 2007, actualité de la recherche en éducation et en formation. http://www.congresintaref.org/actes pdf

Bouillier-Oudot, M.-H., \& Asloum, N. (2004). L'interdisciplinarité dans les formations à visée professionnelle. 14ème congrès international de l'Association Mondiale des Sciences de l'Education. Santiago du Chili, mai. 
Bouillier, M.H., Asloum, N. \& Veyrac, H. (2008). A quels modèles d'apprentissage se réfèrent les enseignants dans des formations à visée professionnelle ? Une étude de cas dans l'enseignement technique agricole, in $\mathrm{Y}$ Lenoir, $\mathrm{P}$. Pastré, (sous la dir). Didactique professionnelle et didactique des disciplines en débat (pp. 189-211). Toulouse: Octarès.

Chatigny, C., \& Veyrac, H. (2005). L'ergonomie au service des pratiques enseignantes Pratiques de formation pour les enseignants de l'enseignement technique et professionnel : questions pour la recherche Journées Franco-Québécoises PATRE - Centre de Recherche sur la Formation Professionnelle -ENFA -Auzeville - 22-24 juin.

DGER (1994). Brevet de technicien Supérieur Agricole Aménagements paysagers, Ministère de l'Agriculture et de la pêche, consulté le 15 février 2013

http://www.chlorofil.fr/index.php?elD=tx_nawsecuredl\&u=0\&file=fileadmin/user_upload/diplom es/ref/btsa/AP/btsa-

ap.pdf\&t=1361021183\&hash=9582e49969480a2e5d8faa911bc2c78d54ccae6d

Leplat, J. (1997). Regards sur l' activité en situation de travail Contribution à la psychologie ergonomique. Paris : PUF.

Mayen, P. (2007). Passer du principe d'alternance à l'usage de l'expérience en situation de travail comme moyen de formalisation et de professionnalisation, In F. Mehran, C. Ronveaux \& S. Vanhulle, Alternances en formation, (pp 83-100) Raisons éducatives, Bruxelles : De Boeck.

Pastré, P. (2008) Apprentissage et activité, In Y. Lenoir \& P. Pastré (Eds.), Didactique professionnelle et didactiques disciplinaires en débat (pp. 53-79). Toulouse : Octares.

Veyrac, H. (2005). Vers une analyse des pratiques enseignantes d'accompagnement d'élèves en stages en entreprise, 5ème Colloque International Recherche(s) et Formation " Former des enseignants-professionnels, savoirs et compétences », Nantes, 14-15-16 février 2005 [CDROM].

Veyrac, H. (2006). Analyse d'une activité d'explicitation des expériences préprofessionnelles des élèves en classe, Biennale de l'éducation, Expérience(s), savoir(s), sujet(s), Lyon, France, 1114 avril.

Consulté le 15 février 2013 http://www.inrp.fr/biennale/8biennale/contrib/affich.php?\&num=337

Veyrac, H. \& Asloum, N. (2009). Les tâches appropriées des professeurs d'enseignement professionnel. Illustration du hiatus entre travail en entreprise et formation. Activités, 6 (1), pp. 69-86. http://www.activites.org/v6n1/v6n1.pdf

Veyrac, H. \& Bouillier, M.H. (2011). Les concepts de représentations de la tâche en ergonomie pour l'analyse des fondements des pratiques professionnelles des enseignants, in Ph. Maubant, \& S. Martineau (Eds). Pour une lecture compréhensive des fondements des savoirs professionnels des enseignants. (pp. 219-242) Ottawa : Presses de l'université d'Ottawa. 